\title{
New generalizations of modular spaces
}

\author{
Tayebe Lal Shateri (iD \\ Department of Mathematics and Computer Sciences, Hakim Sabzevari University, Sabzevar, P.O. Box \\ 397 , Iran
}

\begin{abstract}
In the present paper, we introduce the concept of $\mathcal{F}$-modular, which is a generalization of the modular notion. Moreover, we introduce a $K_{p}$-modular and $K$-modular, and then compare these concepts together. Finally, we give a characterization of $\mathcal{F}$-modulars.
\end{abstract}

Mathematics Subject Classification (2010). 46A 80

Keywords. modular space, $\mathcal{F}$-modular space, $K$-modular space

\section{Introduction}

A modular on a space $\mathcal{X}$ is a mapping $\rho: \mathcal{X} \rightarrow[0, \infty]$ satisfying the following properties:

(i) $\rho(x)=0$ if and only if $x=0$,

(ii) $\rho(\alpha x)=\rho(x)$ for every scaler $\alpha$ with $|\alpha|=1$,

(iii) $\rho(\alpha x+\beta y) \leq \rho(x)+\rho(y)$ for every $\alpha, \beta \geq 0$ such that $\alpha+\beta=1$.

A modular $\rho$ defines a corresponding modular space, i.e., the vector space $X_{\rho}$ given by

$$
X_{\rho}=\{x \in \mathcal{X}: \quad \rho(\lambda x) \rightarrow 0 \text { as } \lambda \rightarrow 0\} .
$$

The theory of modular spaces was founded by Nakano [15] and was intensively developed by Luxemburg [10], Koshi and Shimogaki [8] and Yamamuro [18] and their collaborators. In the present time the theory of modulars and modular spaces is extensively applied, in particular, in the study of various Orlicz spaces [16] and interpolation theory $[9,12]$, which in their turn have broad applications [13]. Shateri [17] introduced the notion of a $C^{*}$-valued modular, and investigated some fixed point theroems in such spaces.

Recently, many interesting extentions of the metric space appeared. The notion of a $b$ metric space introduced by Czerwik [2]. Fagin, et al. [3] introduced $s$-relaxed $\operatorname{metric}$ spaces. Gähler [4] defined the notion of a 2-metric on the product set $X \times X \times X$. The reader can see more generalizations of the notion of a metric space in $[1,5,7,12,14]$. Jleli and Samet [6] introduced the $\mathcal{F}$-metric concept. They defined a natural topology in such spaces, and studied their topological properties.

In this paper, by using some ideas of [6] we introduce the $\mathcal{F}$-modular concept, which is a generalization of the modular space notion. We prove that any modular is an $\mathcal{F}$-modular, but the converse is not true in general, which shows that our concept is more general than the standard modular concept.

Email address: t.shateri@hsu.ac.ir

Received: 24.02.2019; Accepted: 08.08.2019 
Moreover, we introduce a $K_{p}$-modular and $K$-modular, and then compare these concepts together. Finally, we introduce the notion of $\mathcal{F}$-modulars boundedness, which is used to provide a characterization of $\mathcal{F}$-modular spaces.

\section{2. $\mathcal{F}$-modulars}

We start by introducing the following set which plays an important role in our topic. Let $\mathcal{F}$ be the set of all functions $f:(0,+\infty) \rightarrow \mathbb{R}$ which satisfy in the following conditions

$\left(\mathcal{F}_{1}\right) f$ is non-decreasing,

$\left(\mathcal{F}_{2}\right)$ For every sequence $\left\{t_{n}\right\}$ in $(0,+\infty), \lim _{n \rightarrow+\infty} t_{n}=0$ if and only if $\lim _{n \rightarrow+\infty} f\left(t_{n}\right)=-\infty$.

Now we define a new concept of a modular space.

Definition 2.1. Let $X$ be a linear space, and let $\delta: X \rightarrow[0,+\infty)$ be a given mapping. Suppose there exists $(f, \gamma) \in \mathcal{F} \times[0,+\infty)$ such that

$\left(\delta_{1}\right) \delta(x)=0$ if and only if $x=0$,

$\left(\delta_{2}\right) \delta(\alpha x)=\delta(x)$ for every scaler $\alpha$ with $|\alpha|=1$,

$\left(\delta_{3}\right)$ For each $x, y \in \mathcal{X}$, for each $2 \leq n \in \mathbb{N}$, and for every $\left\{u_{i}\right\}_{i=1}^{n}$ in $\mathcal{X}$ with $u_{1}=x$ and $u_{n}=y$, if $\delta(\alpha x+\beta y)>0$ for $\alpha, \beta>0$ in which $\alpha+\beta=1$,

then

$$
f(\delta(\alpha x+\beta y)) \leq f\left(\sum_{i=1}^{n} \delta\left(u_{i}\right)\right)+\gamma
$$

Then $\delta$ is called an $\mathcal{F}$-modular on $\mathcal{X}$, and the pair $(\mathcal{X}, \delta)$ is said to be an $\mathcal{F}$-modular space.

Note that if $\rho$ is a modular on $\mathcal{X}$, then it is an $\mathcal{F}$-modular with $f(t)=\ln t$ and $\gamma=0$. Clearly $\left(\delta_{1}\right)$ and $\left(\delta_{2}\right)$ satisfied. On the other hand, for each $x, y \in \mathcal{X}$, for every $2 \leq n \in \mathbb{N}$, and for every $\left\{u_{i}\right\}_{i=1}^{n}$ in $\mathcal{X}$ with $u_{1}=x$ and $u_{n}=y$, we have

$$
\ln (\rho(\alpha x+\beta y)) \leq \ln (\rho(x)+\rho(y)) \leq \ln \left(\sum_{i=1}^{n} \rho\left(u_{i}\right)\right),
$$

for $\alpha, \beta>0$ in which $\alpha+\beta=1$.

In the following example we give an $\mathcal{F}$-modular space which is not a modular space.

Example 2.2. Let $X=[1, \infty)$, define the mapping $\delta: X \rightarrow[0,+\infty)$ as follows

$$
\delta(x)= \begin{cases}x^{2} & x \in[1,2), \\ x & x \geq 2,\end{cases}
$$

for all $x \in \mathcal{X}$. Then $\delta$ is not a modular. Indeed, $\delta$ does not satisfy ( $i i i)$, because for $x=1$, $y=2, \alpha=\frac{1}{5}$ and $\beta=\frac{4}{5}$, we get

$$
\delta(\alpha x+\beta y)=\delta\left(\frac{1}{5}+\frac{8}{5}\right)=\delta\left(\frac{9}{5}\right)=\frac{81}{25}>\delta(x)+\delta(y)=3 .
$$

Now, we show that $\delta$ is an $\mathcal{F}$-modular. Fix $x, y \in X$, and let $\left\{u_{i}\right\}_{i=1}^{n}$ in $\mathcal{X}$ with $u_{1}=x$ and $u_{n}=y$. Put $I=\left\{i=1, \ldots, n ; u_{i} \in[1,2)\right\}$ and $J=\{1,2, \ldots\}-I$, then we have

$$
\sum_{i=1}^{n} \delta\left(u_{i}\right)=\sum_{i \in I} \delta\left(u_{i}\right)+\sum_{j \in J} \delta\left(u_{j}\right)=\sum_{i \in I} u_{i}^{2}+\sum_{j \in J} u_{j} .
$$


Now we have two cases.

Case 1: If $\alpha x+\beta y \notin[1,2)$, then

$$
\begin{aligned}
\delta(\alpha x+\beta y) & =\alpha x+\beta y \\
& \leq x+y \leq \sum_{i=1}^{n} u_{i}=\sum_{i \in I} u_{i}+\sum_{j \in J} u_{j} \\
& \leq \sum_{i \in I} u_{i}^{2}+\sum_{j \in J} u_{j} \\
& =\sum_{i=1}^{n} \delta\left(u_{i}\right) .
\end{aligned}
$$

Case 2: If $\alpha x+\beta y \in[1,2)$, then we have

$$
\begin{aligned}
\delta(\alpha x+\beta y) & =(\alpha x+\beta y)^{2} \\
& \leq 2(\alpha x+\beta y) \\
& \leq 2(x+y) \\
& \leq 2\left(\sum_{i \in I} u_{i}+\sum_{j \in J} u_{j}\right) \\
& \leq 2\left(\sum_{i \in I} u_{i}^{2}+\sum_{j \in J} u_{j}\right) \\
& =2 \sum_{i=1}^{n} \delta\left(u_{i}\right) .
\end{aligned}
$$

The above cases show that $\delta$ satisfies $\left(\delta_{3}\right)$ with $f(t)=\ln t, t>0$ and $\gamma=\ln 2$. Therefore $\delta$ is an $\mathcal{F}$-modular.

Now,we define a $K_{p}$-modular on a space $\mathcal{X}$, also we provide an example of an $\mathcal{F}$-modular space that cannot be an $K_{p}$-modular space, which confirms that the class of $\mathcal{F}$-modular spaces is more large than the class of $K_{p}$-modular spaces.

Definition 2.3. Let $\Delta: X \rightarrow[0,+\infty)$ be a mapping satisfies $\left(\delta_{1}\right),\left(\delta_{2}\right)$, and $\left(\Delta_{3}\right)$ There exists $K \geq 1$ such that for every $x, y \in \mathcal{X}$, for every $2 \leq n \in \mathbb{N}$, for every $\left\{u_{i}\right\}_{i=1}^{n}$ in $X$ with $u_{1}=x$ and $u_{n}=y$, we have

$$
\Delta(\alpha x+\beta y) \leq K\left(\sum_{i=1}^{n} \Delta\left(u_{i}\right)\right),
$$

for $\alpha, \beta>0$ in which $\alpha+\beta=1$. Then $\Delta$ is called a $K_{p}$-modular, and $(X, \Delta)$ is said to be a $K_{p}$-modular space.

It is clear that $\Delta$ satisfies $\left(\delta_{3}\right)$ with $f(t)=\ln t, t>0$ and $\gamma=\ln K$, and hence $\Delta$ is an $\mathcal{F}$-modular. Notice that the mapping $\delta$ in Example 2.2 satisfies in $\left(\Delta_{3}\right)$ with $K=2$. The following example shows that the class of $\mathcal{F}$-modulars is more large than the class of $K_{p}$ modulars.

Example 2.4. Let $X=\mathbb{Z}$. Define the mapping $\delta: X \rightarrow[0,+\infty)$ by

$$
\delta(x)= \begin{cases}\frac{1}{e^{|x|}} & x \neq 0, \\ 0 & x=0,\end{cases}
$$

for all $x \in \mathcal{X}$. Then $\delta$ is a $\mathcal{F}$-modular. It is clear that $\delta$ satisfies $\left(\delta_{1}\right)$ and $\left(\delta_{2}\right)$. In order to check $\left(\delta_{3}\right)$, let

$$
f(t)=-\frac{1}{t}, \quad(t>0)
$$


It can be easily seen that $f \in \mathcal{F}$. Fix $x, y \in X$ and $\alpha, \beta>0$ in which $\alpha+\beta=1$ with $\delta(\alpha x+\beta y)>0$. For every $n \in \mathbb{N}$, and for every $\left\{u_{i}\right\}$ in $X$ with $u_{1}=x$ and $u_{2}=y$, we have

$$
\begin{aligned}
1 & +f\left(\sum_{i=1}^{n} \delta\left(u_{i}\right)\right)-f(\delta(x)+\delta(y)) \\
& =1-\frac{1}{\sum_{i=1}^{n} \frac{1}{e^{\left|u_{i}\right|}}}+\frac{1}{\frac{1}{e^{|\alpha x+\beta y|}}} \\
& =1-\frac{1}{\sum_{i=1}^{n} \frac{1}{e^{\left|u_{i}\right|}}}+e^{|\alpha x+\beta y|} \\
& >1+1+e^{|\alpha x+\beta y|} \\
& \geq 0 .
\end{aligned}
$$

Note that the first inequality holds because $\sum_{i=1}^{n} \frac{1}{e^{\left|u_{i}\right|}}>0>-1$ and so $-\frac{1}{\sum_{i=1}^{n} \frac{1}{e^{\left|u_{i}\right|}}}>1$.

Therefore we get

$$
f(\delta(x)+\delta(y)) \leq f\left(\sum_{i=1}^{n} \delta\left(u_{i}\right)\right)+1 .
$$

Consequently $\delta$ is an $\mathcal{F}$-modular.

Next, we shall prove $\delta$ is not a $K_{p}$-modular. Suppose that $\delta$ satifies $\left(\Delta_{3}\right)$ with a certain $K \geq 1$. Consider $u_{1}=x=4 n, u_{2}=y=0$ and $\alpha=\beta=\frac{1}{2}$. Then we have

$$
\delta(\alpha x+\beta y)=\delta(2 n) \leq K(\delta(x)+\delta(y))=K \delta(4 n),
$$

this implies that

$$
e^{2 n} \leq K
$$

Passing to the limit as $n \rightarrow+\infty$, we obtain a contradiction. Therefore, $\delta$ can not be a $K_{p}$-modular.

In following, we introduce another concept of a modular space which is more large than the class of $\mathcal{F}$-modular spaces and $K_{p}$-modular spaces.

Definition 2.5. Let $X$ be a linear space, and let $\rho: X \rightarrow[0,+\infty)$ be a mapping. Let there exists $K \geq 1$ such that

$\left(\rho_{1}\right) \rho(x)=0$ if and only if $x=0$,

$\left(\rho_{2}\right) \rho(\alpha x)=\rho(x)$ for every scalar $\alpha$ with $|\alpha|=1$,

$\left(\rho_{3}\right) \rho(\alpha x+\beta y) \leq K(\rho(x)+\rho(y))$, for $\alpha, \beta>0$ in which $\alpha+\beta=1$.

Then $\rho$ is called a $K$-modular.

Notice that, each modular is a $K$-modular with $K=1$. Also every $K_{p}$-modular is a $K$-modular. In following we give an example that shows the converse is not true in general.

Example 2.6. Let $X=[0,1]$, and let $\delta: X \rightarrow[0,+\infty)$ be the mapping defined by

$$
\delta(x)= \begin{cases}x^{2} & x \in[0,1) \\ 0 & x=1\end{cases}
$$

It can be easily seen that $\delta$ is a $K$-modular with $K=2$. Next, we prove that $\delta$ is not an $\mathcal{F}$-modular. Suppose that there exists $(f, \gamma)$ such that $\delta$ satisfies $\left(\delta_{3}\right)$. Let $n \in \mathbb{N}$, and put

$$
x=u_{1}=0, y=u_{n}=1, u_{i}=\frac{1}{n}, \quad i=2, \ldots, n-1 .
$$

Then for $\alpha=\beta=\frac{1}{2},\left(\delta_{3}\right)$ implies that

$$
f\left(\delta\left(\frac{x}{2}+\frac{y}{2}\right)\right) \leq f\left(\delta\left(u_{1}\right)+\delta\left(u_{2}\right)+\cdots+\delta\left(u_{n-1}\right)+\delta\left(u_{n}\right)\right)+\gamma .
$$


Hence

By $\left(\mathcal{F}_{2}\right)$, we have

$$
f\left(\frac{1}{2}\right)=\frac{1}{4} \leq f\left(\frac{n-2}{n^{2}}\right)+\gamma .
$$

$$
\lim _{n \rightarrow+\infty} f\left(\frac{n-2}{n^{2}}\right)+\gamma=-\infty,
$$

which is a contradiction. Consequently $\delta$ is not an $\mathcal{F}$-modular.

Moreover $\delta$ is not a $K_{p}$-modular. Infact if $\delta$ satisfies $\left(\Delta_{3}\right)$, and let

$$
x=u_{1}=0, y=u_{n}=1, u_{i}=\frac{1}{n}, \quad i=2, \ldots, n-1,
$$

then for $\alpha=\beta=\frac{1}{2}$, by $\left(\Delta_{3}\right)$ we conclude that

$$
\delta\left(\frac{x}{2}+\frac{y}{2}\right) \leq \delta\left(u_{1}\right)+\delta\left(u_{2}\right)+\cdots+\delta\left(u_{n-1}\right)+\delta\left(u_{n}\right)+\gamma .
$$

Therefore

By $\left(\mathcal{F}_{2}\right)$, we have

$$
\frac{1}{2}=\frac{1}{4} \leq \frac{n-2}{n^{2}}
$$

$$
\lim _{n \rightarrow+\infty} \frac{n-2}{n^{2}}=0
$$

which is a contradiction.

Remark 2.7. One can easily see that the mapping $\delta$ defined by (2.1) in Example 2.4, is not also a $K$-modular on $X$.

\section{F-modular boundedness}

In this section, we introduce the concept of $\mathcal{F}$-modular boundedness, which is used to provide a characterization of $\mathcal{F}$-modular spaces.

Definition 3.1. Let $X$ be a linear space, and let $\delta: X \rightarrow[0,+\infty)$ be a mapping satisfying $\left(\delta_{1}\right)$ and $\left(\delta_{2}\right)$. We call the pair $(\mathcal{X}, \delta)$ is $\mathcal{F}$-modular bounded with respect to $(f, \gamma) \in$ $\mathcal{F} \times[0,+\infty)$, if there exists a modular $\rho$ on $\mathcal{X}$ such that for every $x, y \in \mathcal{X}$, and for $\alpha, \beta>0$ in which $\alpha+\beta=1, \delta(\alpha x+\beta y)>0$ implies that

$$
f(\rho(\alpha x+\beta y)) \leq f(\delta(x)+\delta(y)) \text { and } f(\delta(\alpha x+\beta y)) \leq f(\rho(\alpha x+\beta y))+\gamma .
$$

We can prove the following result.

Theorem 3.2. Let $X$ be a space and let $\delta: X \rightarrow[0,+\infty)$ be a mapping satisfying $\left(\delta_{1}\right)$ and $\left(\delta_{2}\right)$. Let $(f, \gamma) \in \mathcal{F} \times[0,+\infty)$ such that $f$ is continuous from the right. Then the followings are equivalent:

(i) $(X, \delta)$ is an $\mathcal{F}$-modular on $\mathcal{X}$ with $(f, \gamma)$ given above.

(ii) $(\mathcal{X}, \delta)$ is an $\mathcal{F}$-modular bounded with respect to $(f, \gamma)$.

Proof. Suppose that $(X, \delta)$ is an $\mathcal{F}$-modular on $X$ with respect to $(f, \gamma)$. We define the mapping $\rho: X \rightarrow[0,+\infty)$ by

$$
\rho(\alpha x+\beta y)=\inf \left\{\sum_{i=1}^{n} \delta\left(u_{i}\right): n \in \mathbb{N}, n \geq 2,\left(u_{i}\right)_{i=1}^{n} \subset X, u_{1}=x, u_{n}=y\right\},
$$

for all $x, y \in X$ and for $\alpha, \beta>0$ in which $\alpha+\beta=1$. We show that $\rho$ is a modular on $X$. Note that

$$
\rho(x)=\inf \left\{\sum_{i=1}^{n} \delta\left(u_{i}\right): n \in \mathbb{N}, n \geq 2,\left(u_{i}\right)_{i=1}^{n} \subset X, u_{1}=u_{n}=x\right\}
$$


so if $x=0$, then $\rho(x)=0$. Since $\delta(\alpha x)=\delta(x)$, for each $\alpha$ such that $|\alpha|=1$, it follows from the definition of $\rho$ that

$$
\rho(\alpha x)=\rho(x), \quad x \in \mathcal{X} .
$$

Now, let $x \in \mathcal{X}$ be such that $\rho(x)=0$. Suppose that $x \neq 0$. Given $\varepsilon>0$, then there exists $n \in \mathbb{N}, n \geq 2$, and $\left(u_{i}\right)_{i=1}^{n} \subset \mathcal{X}$ with $u_{1}=u_{n}=x$ such that

$$
\sum_{i=1}^{n} \delta\left(u_{i}\right)<\varepsilon
$$

By $\left(\mathcal{F}_{1}\right)$, we obtain

$$
f\left(\sum_{i=1}^{n} \delta\left(u_{i}\right)\right) \leq f(\varepsilon)
$$

More over, putting $y=x$ in $\left(\delta_{3}\right)$ deduce that

$$
f(\delta(x)) \leq f\left(\sum_{i=1}^{n} \delta\left(u_{i}\right)\right)+\gamma
$$

Using (3.2) and (3.3), we get

$$
f(\delta(x)) \leq f(\varepsilon)+\gamma
$$

By $\left(\mathcal{F}_{2}\right)$, we obtain

$$
\lim _{\varepsilon \rightarrow 0^{+}}(f(\varepsilon)+\gamma)=-\infty
$$

which is a contradiction. Now, let $x, y \in X$ and let $\alpha, \beta>0$ be such that $\alpha+\beta=1$. Suppose $\varepsilon>0$ is arbitrary. Then by definition of $\rho$, there exist $\left\{u_{i}\right\}_{i=1}^{n}$ and $\left\{v_{j}\right\}_{j=1}^{m}$ in $X$ such that $u_{1}=u_{n}=x, v_{1}=v_{m}=y$, and

$$
\sum_{i=1}^{n} \delta\left(u_{i}\right)<\rho(x)+\varepsilon, \quad \sum_{j=1}^{m} \delta\left(v_{j}\right)<\rho(y)+\varepsilon .
$$

Put $w_{1}=u_{1}=x$, and $w_{i}=u_{i}$ for every $2 \leq i \leq n, w_{i}=v_{n+m-i-1}$ for every $n+1 \leq i \leq$ $n+m-1$, and $w_{n+m}=u_{m}=y$. Then we obtain

$$
\begin{aligned}
\rho(\alpha x+\beta y) & \leq \sum_{i=1}^{n+m} \delta\left(w_{i}\right) \\
& =\sum_{i=1}^{n} \delta\left(u_{i}\right)+\sum_{j=1}^{m} \delta\left(v_{j}\right) \\
& <\rho(x)+\rho(y)+2 \varepsilon .
\end{aligned}
$$

Passing to the limit as $\varepsilon \rightarrow 0^{+}$, we get

$$
\rho(\alpha x+\beta y) \leq \rho(x)+\rho(y) .
$$

Now, we shall prove that $\delta$ satisfies (3.1). For this, let $x, y \in \mathcal{X}$, and for $\alpha, \beta>0$ in which $\alpha+\beta=1, \delta(\alpha x+\beta y)>0$. It is clear that

$$
\rho(\alpha x+\beta y) \leq \delta(x)+\delta(y)
$$

and $\left(\mathcal{F}_{1}\right)$ implies that

$$
f(\rho(\alpha x+\beta y)) \leq f(\delta(x)+\delta(y)) .
$$

Let $\varepsilon>0$. Then, there exist $n \in \mathbb{N}$, and $\left\{u_{i}\right\}_{i=1}^{n} \subset \mathcal{X}$ with $u_{1}=x$ and $u_{n}=y$ such that

$$
\sum_{i=1}^{n} \delta\left(u_{i}\right)<\rho(\alpha x+\beta y)+\varepsilon
$$


Hence

$$
f\left(\sum_{i=1}^{n} \delta\left(u_{i}\right)\right) \leq f(\rho(\alpha x+\beta y)+\varepsilon)
$$

By $\left(\delta_{3}\right)$, we obtain

$$
f(\delta(\alpha x+\beta y) \leq f(\rho(\alpha x+\beta y)+\varepsilon)+\gamma .
$$

Passing to the limit as $\varepsilon \rightarrow 0^{+}$, and using the right continuity of $f$, we get

$$
f(\delta(\alpha x+\beta y) \leq f(\rho(\alpha x+\beta y))+\gamma .
$$

We deduce from (3.4) and (3.5) that

$$
f(\rho(\alpha x+\beta y)) \leq f(\delta(x)+\delta(y)) \leq f(\rho(\alpha x+\beta y))+\gamma .
$$

Therefore $(X, \delta)$ is $\mathcal{F}$-modular bounded with respect to $(f, \gamma)$.

Now, let $(\mathcal{X}, \delta)$ is $\mathcal{F}$-modular bounded with respect to $(f, \gamma)$, that is, there exists a modular $\rho$ on $X$ such that (3.1) satisfied. Let $x, y \in X$, and let $\alpha, \beta>0$ be such that $\alpha+\beta=1$, and $\delta(\alpha x+\beta y)>0$. Suppose $n \in \mathbb{N}$, and $\left\{u_{i}\right\}_{i=1}^{n}$ with $u_{1}=x, u_{n}=y$. Since $\rho$ is a modular, we have

$$
\rho(\alpha x+\beta y) \leq \rho(x)+\rho(y) \leq \sum_{i=1}^{n} \rho\left(u_{i}\right) .
$$

Using $\left(\mathcal{F}_{1}\right)$ and the fact that if $\delta(x)+\delta(y)>0$, and

$$
f(\rho(\alpha x+\beta y)) \leq f(\delta(x)+\delta(y)),
$$

we deduce that

$$
\rho(\alpha x+\beta y) \leq \delta(x)+\delta(y)
$$

By (3.6) and (3.7), we get

$$
f(\rho(\alpha x+\beta y)) \leq f(\delta(x)+\delta(y)) \text { and } f(\delta(\alpha x+\beta y)) \leq f(\rho(\alpha x+\beta y))+\gamma .
$$

By $\left(\mathcal{F}_{1}\right)$ we deduce that

$$
f(\rho(\alpha x+\beta y))+\gamma \leq f\left(\sum_{i=1}^{n} \delta\left(u_{i}\right)\right)+\gamma
$$

On the other hand

$$
f(\delta(\alpha x+\beta y)) \leq f(\rho(\alpha x+\beta y))+\gamma
$$

we conclude that

$$
f(\delta(\alpha x+\beta y)) \leq f\left(\sum_{i=1}^{n} \delta\left(u_{i}\right)\right)+\gamma
$$

Therefore, $\delta$ is an $\mathcal{F}$-modular on $\mathcal{X}$.

Theorem 3.2 gives a characterization of $\mathcal{F}$-modulars as follows.

Corollary 3.3. An $\mathcal{F}$-modular on a space $\mathcal{X}$ is an $\mathcal{F}$-modular bounded mapping.

Remark 3.4. Note that in the proof of Theorem 3.2, the right continuity assumption of $f$ is used only to prove that $(i) \Rightarrow(i i)$. However, $(i i) \Rightarrow(i)$ holds for any $f \in \mathcal{F}$. 


\section{References}

[1] A. Branciari, A fixed point theorem of Banach-Caccioppoli type on a class of generalized metric spaces, Publ. Math. Debrecen 57, 31-37, 2000.

[2] S. Czerwik, Contraction mappings in b-metric spaces, Acta Math. Univ. Ostrav. 1 (1), 511, 1993.

[3] R. Fagin, R. Kumar and D. Sivakumar, Comparing top k lists, SIAM J. Discrete Math. 17 (1), 134-160, 2003.

[4] V.S. Gähler, 2-metrische Räume und ihre topologische struktur, Math. Nachr. 26, $115-118,1963 / 1964$.

[5] M. Jleli and B. Samet, A generalized metric space and related fixed point theorems, Fixed Point Theory Appl. 2015, 14 pages, 2015.

[6] M. Jleli and B. Samet, On a new generalization of metric spaces, Fixed Point Theory Appl. 20 (3), 20 pages, 2018.

[7] R. Kopperman and H. Pajoohesh, Generalizations of metrics and partial metrics, Hacet. J. Math. Stat. 46 (1), 9-14, 2017.

[8] S. Koshi and T. Shimogaki, On F-norms of quasi-modular spaces, J. Fac. Sci. Hokkaido Univ. Ser. I. 15 (3), 202-218, 1961.

[9] M.A. Krasnoselskii and Y.B. Rutickii, Convex functions and Orlicz spaces (in Russian), Fizmatgiz, Moskva, 1958; Translated by L.F. Boron, Noordhoff, Groningen, 1961.

[10] W.A. Luxemburg, Banach function spaces, Ph. D. Thesis, Delft University of Technology, Delft, The Netherlands, 1959.

[11] L. Maligranda, Orlicz Spaces and Interpolation, in: Seminars in Math. 5, Univ. of Campinas, Brazil, 1989.

[12] S.G. Matthews, Partial metric topology, Proc. 8th Summer Conference on General Topology and Applications, Ann. New York Acad. Sci. 728, 183-197, 1994.

[13] J. Musielak, Orlicz Spaces and Modular Spaces, in: Lecture Notes in Math. 1034, Springer-Verlag, Berlin, 1983.

[14] Z. Mustafa and B. Sims, A new approach to generalized metric spaces, J. Nonlinear Convex Anal. 7 (2), 289-297, 2006.

[15] H. Nakano, Modulared Semi-Ordered Linear Spaces, in: Tokyo Math. Book Ser. 1, Maruzen Co., Tokyo, 1950.

[16] W. Orlicz, Collected Papers, Vols. I, II, PWN, Warszawa, 1988.

[17] T.L. Shateri, $C^{*}$-algebra-valued modular spaces and fixed point theorems, J. Fixed Point Theory Appl. 19 (2), 1551-1560, 2017.

[18] S. Yamamuro, On conjugate spaces of Nakano spaces, Trans. Amer. Math. Soc. 90, 291-311, 1959. 